\title{
De la mécanique au sens : le cas du pronom en en français ${ }^{1}$
}

\author{
Ihsane, Tabea, Forel, Claire, \& Kusseling, Françoise \\ Université de Genève et Brigham Young University \\ Tabea.Ihsane@unige.ch, Claire.Forel@unige.ch, fsk@byu.edu
}

\section{Introduction}

Ce texte met l'accent sur le pronom clitique en français illustré dans l'exemple (1) et sur la façon dont il est présenté dans les manuels ou les grammaires universitaires proposés aux niveaux intermédiaire à avancé pour locuteurs non-natifs du français, en particulier pour anglophones (français langue étrangère, FLE).

(1) Jean vient de Paris. Il en vient.

Dans (1), en pronominalise le constituant en gras de Paris. De manière plus générale, ce pronom peut remplacer les constituants introduits par de (2), ceux qui suivent un nombre (3) ou une expression de quantité (4).

(2) Jean a mangé de la tarte. Il en a mangé.

(3) Jean a acheté quatre livres. Il en a acheté quatre.

(4) Jean a acheté beaucoup de livres. Il en a acheté beaucoup.

Les manuels d'acquisition du FLE pour les anglophones abordent généralement l'utilisation de en comme dans les exemples ci-dessus, en fournissant une liste d'expressions qui peuvent être pronominalisées par en. C'est ce que nous observons dans les différents manuels examinés dans le présent article. En d'autres termes, ces ouvrages proposent de remplacer une forme donnée par une autre, en, d'une manière très mécanique. Comme en est une pro-forme, c'est-à-dire un pronom remplaçant un plus grand constituant, une telle méthode n'est pas surprenante et semble logique, car elle permet à l'orateur d'éviter les répétitions. Cependant, nous montrons dans cet article que cette approche est problématique car elle omet de décrire des faits cruciaux. Nous y examinons en particulier deux cas, très fréquents en français courant, qui ne sont pas couverts par les règles de substitution figurant dans les manuels : les 
constituants introduits par des et les constituants formés de de + substantif sans déterminant, que nous appellerons 'nom nu'.

Comme nous le verrons, les constituants avec des ne peuvent pas toujours être remplacés par en, et quand ils le peuvent, deux interprétations différentes sont possibles, selon le contexte (la signification peut être indéfinie ou partitive). La difficulté avec $d e+$ nom nu, est que quand en est utilisé, il doit, dans certains cas mais pas toujours, apparaître accompagné d'un nombre ou d'une expression de quantité. Les règles de substitution données aux apprenants ne leur permettent pas de distinguer ces contextes.

Ce que nous montrons dans cet article est que ces questions peuvent être résolues si certains aspects liés au concept de référence, à ne pas confondre avec les référents dans un monde (réel ou imaginaire), sont expliqués à l'apprenant. Cela implique de tenir compte du contexte dans lequel en est utilisé. Plus généralement, nous soulignons le fait que les règles de transformation sont limitées parce qu'elles ne se concentrent que sur les aspects morphologiques sans tenir compte du contexte, dans lequel la référence est accessible.

La notion de référence nécessaire à l'enseignement de en peut être expliquée de manière simple sans introduire des idées théoriques complexes, car elle consiste essentiellement en l'utilisation et la mise en relation de notions qui devraient être familières à l'apprenant, à partir de l'utilisation des déterminants par exemple : la distinction comptable/non-comptable, la relation partie-tout (partitivité) et la différence entre les éléments qui ont été introduits dans le discours/contexte (définis) et des éléments qui ne l'ont pas été (indéfinis). La prise en considération du concept de référence, et donc du contexte de l'énoncé, nous permettra de façon décisive de non seulement démêler les deux interprétations de en dans les contextes avec des, mais aussi de distinguer ces deux usages d'autres contextes dans lesquels le pronom les remplace un constituant avec des. En outre, cela nous permettra d'expliquer les différences entre les expressions $d e+$ noms nus évoquées ci-dessus.

Cet article aborde brièvement d'autres questions comme les constructions négatives et les difficultés liées à l'utilisation de en spécifiques aux locuteurs natifs de l'anglais apprenant le français, et en particulier le fait qu'en n'est parfois pas traduit en anglais.

\section{Les manuels pour les apprenants anglophones du français (FLE)}

Les manuels universitaires de français pour anglophones que nous avons examinés à propos de l'enseignement de en sont les suivants ${ }^{2}$ :

1. Valette et Valette (1989)

2. Thompson et Hirsch (2003)

3. Terrell et al. (2008)

4. Mitschke (2008)

5. Ollivier et Beaudoin (2008)

6. Oates et Dubois (2010)

7. Mitschke et Tano (2010)

Ces manuels sont destinés en majorité à des apprenants de niveau intermédiaire. Il ne s'agit donc pas d'étudiants très avancés dont l'interlangue est suffisamment développée pour qu'ils n'aient pas recours aux règles mécaniques de substitution présentées dans ce genre d'ouvrages. 
Pour expliquer comment en est utilisé, les manuels ci-dessus proposent une liste de formes qui peuvent être remplacées par en. En d'autres termes, l'idée générale est qu'il y a substitution d'une forme par une autre. Par exemple, Oates et Dubois mentionnent qu'en prend la place d'un objet (nom ou infinitif) précédé par une certaine forme de de (2010:100) et Ollivier et Beaudoin signalent qu'en remplace de, d', du, de la, de l', des + une chose (2008: 82) (ce qui correspondrait à (1) et (2)). Souvent, les contextes dans lesquels en apparaît sont énumérés plus précisément : la plupart des manuels expliquent que l'objet de la préposition de peut être remplacé par en (Valette et Valette, 1989 : 345 ; Mitschke, 2008 : 175 ; Mitschke et Tano, $2010: 314$ ), parfois sous quelques conditions comme : « si le nom objet est une chose ou un lieu » (Thompson et Hirsch, $2003: 188$ ) ou « le nom d'une chose » (Terrell et al., $2008: 255$ ). Au rang des autres formes qui sont généralement citées comme éléments remplaçables par en figurent les locutions introduites par l'article indéfini et les articles dits partitifs. Certains manuels classent ces articles séparément (Terrell et al. 2008 : 254: les articles partitifs (du, de la, de l'), les articles indéfinis (un, une, des)), tandis que d'autres fournissent une liste unique (Valette et Valette, 1989 : 345: du, de la, de l', des,) ou ne donnent pas de liste du tout (Thompson et Hirsch, $2003: 188$; Mitschke et Tano, $2010: 314-5$ ). Oates et Dubois (2010:100), Mitschke (2008: 175) et Ollivier et Beaudoin (2008: 82) mentionnent les partitifs mais pas les indéfinis. La différence entre un / une et des n'est pas toujours expliquée (Terrell et al., $2008: 254-5)$ : si le nom qui est remplacé par en, est précédé par un / une, ce dernier est maintenu (5). En revanche, des n'est pas répété : il est inclus dans le constituant remplacé par en comme l'illustre l'exemple (6), dans lequel la réponse avec un astérisque (*) est agrammaticale :

(5) Avez-vous un chien ? Oui, j'en ai un.

(6) Avez-vous des chiens ? Oui, j'en ai. / * Oui, j'en ai des.

La distinction entre un / une et des n'est pas toujours explicite. Certains auteurs, par exemple, ne traitent un que comme un nombre (Oates et Dubois, $2010: 100$; Ollivier et Beaudoin, 2008 : 83). En d'autres termes, ils assimilent les exemples avec un / une à un autre contexte dans lequel en est fréquemment utilisé, c'est-à-dire les constructions avec des nombres (cf. (3)). Tous les manuels que nous avons examinés et qui parlent de en avec des nombres mentionnent aussi les expressions de quantité (beaucoup de, peu de...) (cf. (4)). Terrell et al. (2008: 254-5) et Ollivier et Beaudoin (2008: 82-3) ne mentionnent pas explicitement que le nombre et l'expression de quantité utilisés avec en doivent être répétés, contrairement à Thompson et Hirsch (2003: 188), Mitschke (2008: 175), Oates et Dubois (2010 : 100) et Mitschke et Tano (2010:314). Enfin, seuls deux des manuels que nous avons examinés incluent les constructions négatives dans les contextes où l'on trouve en (Valette et Valette («en remplace les OBJETS DIRECTS introduits par le de négatif », 1989: 345) et Mitschke (« dans une phrase négative, le nombre n'est pas conservé », $2008: 175)$ ). Intéressons-nous maintenant à (7) qui illustre une phrase négative, sans nombre :

(7) Jean n'a pas de chien. Il n'en a pas.

En somme, la discussion ci-dessus montre clairement que tous les manuels que nous avons étudiés établissent une liste d'éléments qui peuvent être remplacés par en. Ceci peut être formalisé par les règles de substitution suivantes : 
(8) a. Les constituants introduits par

de, du, de la, de l', des

b. Les constituants précédés par

des nombres et des expressions de quantité

peuvent être remplacés par en

La clause a) en (8) concerne notamment des exemples tels que (1) et (2) alors que celle en b) se rapporte à des cas tels que (3) et (4).

Bien que ces règles soient claires et précises, elles sont problématiques dans certains cas, comme nous le verrons ci-dessous. ${ }^{3}$

\section{Les données qui sont problématiques pour les règles de substitution}

La supposition que (seules) les règles transformationnelles comme (8) traitent de l'usage de en est problématique pour différentes raisons. Notre discussion se concentrera sur deux points. Le premier concerne les constituants introduits par des comme dans (9), et le deuxième les expressions avec de + nom nu comme en (10) et (11). Nous allons voir qu'en fin de compte les deux problèmes sont liés.

(9) Jean a acheté des livres. Il en a acheté.

(10) Combien de viande veux-tu? J'en veux un peu. / * J'en veux. / * J'en veux deux.

(11) Combien de livres veux-tu? * J'en veux un peu. / * J'en veux. / J'en veux deux.

Commençons avec des exemples comme (9). D'après (8a), un constituant introduit par des peut être remplacée par en. Cependant, dans certains contextes, de tels constituants peuvent être remplacés par le pronom les (ce qui contraste avec Valette et Valette qui écrivent que «les pronoms le, la, les sont utilisés pour remplacer un nom objet direct introduit par un article défini, un démonstratif ou un adjectif possessif »). La séquence suivante illustre ce point :

(12) Jean : J'ai vu des biscuits sur la table.

(13) Marie : Oui, je les ai vus aussi.

Le fait qu'un énoncé avec le pronom les comme (13) soit approprié en réponse à la remarque de Jean dans (12) ne signifie pas qu'un exemple avec en soit agrammatical, comme (14) le montre :

(14) Marie : Oui, j'en ai vu aussi. 
(13) et (14) sont tous deux possibles après (12). Cependant, aucun des manuels sur lesquels nous nous sommes penchés ne mentionne ce fait. Pourtant, pour qu'un apprenant du français comprenne comment utiliser en, il/elle devrait être conscient de ces options et du fait que (13) et (14) n'ont pas la même signification. En (13), le pronom les représente les biscuits vus par Jean tandis que dans (14) en peut représenter d'autres biscuits. Cette différence n'est pas anodine si nous voulons communiquer efficacement, et ne peut être saisie que si le discours /contexte d'énonciation est pris en compte.

Pour qu'un apprenant de la langue française réalise et comprenne la différence entre (13) et (14), les règles de substitution en (8) ne sont pas suffisantes. En effet, bien que les règles en (8) prédisent que (14) soit correct, elles ne suggèrent pas que cette possibilité n'est pas exclusive. En d'autres termes, un apprenant appliquant (8) ne sera pas conscient du contraste existant entre (13) et (14) et ne sera donc pas en mesure de comprendre la différence entre les deux, et encore moins de déterminer à quelle interprétation en correspond. La question est encore plus complexe si l'on considère un exemple comme (15), suivi de la remarque en (16):

(15) Jean : J'ai pris des biscuits qui étaient sur la table.

(16) Marie : Oui, j'en ai pris aussi.

Un énoncé comme (16), qui contient en, diffère de (14) en ce qu'il a un sens partitif : en correspond à une partie des biscuits mentionnés par Jean (relation partie-tout). Encore une fois, la différence entre ces contextes doit être rendue explicite pour qu'un apprenant puisse comprendre comment utiliser en correctement et communiquer efficacement. Bien que dans (14) et dans (16) en remplace un constituant introduit par des, l'utilisation / la signification de en y diffère et cela devrait être signalé à un apprenant.

On trouve les mêmes différences de sens dans les exemples avec des nombres. Considérons la phrase (17), et les réactions qu'elle suscite en (18a-c):

(17) Jean : J'ai acheté 5 pommes.
a. Marie : Oui, je les ai vues.
b. Marie : Moi aussi, j'en ai acheté cinq.
c. Marie : J'ai remarqué. J'en ai mangé deux.

En (18a), les représente toutes les pommes achetées par Jean, en b, en représente cinq pommes différentes et en c en représente un sous-ensemble des cinq pommes achetées par Jean. Des contextes comme (17), contrairement aux constituants introduits par des, conservent le nombre quand en est utilisé (voir (8b)).

Passons maintenant au second point problématique pour les règles de transformation en (8), et qui concerne des exemples comme (10) et (11). Fondamentalement, la première partie de ces exemples, c'està-dire la question, semble similaire (Combien de viande / de livres veux-tu ?). Cependant, la réponse doit être différente : en (10), J'en veux un peu est correct, alors que J'en veux deux ne l'est pas, d'où l'astérisque. En revanche, dans (11), J'en veux deux est grammatical, alors que J'en veux un peu ne l'est pas. Dans les deux cas, on ne peut pas dire J'en veux. Un manuel devrait permettre à l'apprenant de répondre correctement aux questions introduites par combien.

Selon (8a), en peut remplacer le constituant introduit par de dans les exemples évoqués ci-dessus : en (10), il peut pronominaliser de viande et en (11) de livres. Cependant, cette règle est trop simple car de 
telles constructions requièrent la présence d'une quantité explicite comme un peu ou d'un nombre explicite comme deux dans la phrase avec en. Comme cette contrainte n'est pas mentionnée par (8), il est difficile pour l'apprenant de produire, sur la base de ces règles, des réponses appropriées telles que $J^{\prime} e n$ veux un peu et J'en veux deux comme dans (10) et (11) respectivement.

Une première difficulté pour l'apprenant serait donc de comprendre que, dans certains contextes, il ne suffit pas de remplacer une forme de par en parce que du matériel supplémentaire est nécessaire (une quantité ou un nombre). Cela implique bien sûr d'identifier ces contextes linguistiques. Une deuxième difficulté serait de réaliser que des exemples comme (10) et (11) diffèrent des constructions négatives comme (7): même si dans les deux cas de est suivi d'un nom nu, cet exemple-ci ne requière pas une quantité / un nombre explicite pour être grammatical, contrairement à ceux-là. En d'autres termes, sur la base des règles de substitution en (8), il est impossible pour l'apprenant de déterminer : i) si du matériel supplémentaire est requis avec en ou non, et ii) si c'est un nombre ou une expression de quantité qui doit être présent dans la phrase pour qu'elle soit grammaticale. Pour qu'un enseignement de la grammaire soit efficace, ces questions devraient être examinées et expliquées.

En bref, les règles de substitution comme (8) sont limitées, car elles ne tiennent pas compte des données dont il a été question ci-dessus, qui représentent des aspects importants du français. Pour que de tels exemples soient décrits efficacement, il faudrait soit affiner ces règles, soit mettre en avant une autre méthodologie. Dans cet article, nous avons choisi la deuxième option. Nous proposons de prendre une certaine distance vis-à-vis des règles de substitution et d'introduire des concepts plus larges qui permettront à l'apprenant de mettre en relation les données présentées ci-dessus et de différencier les diverses interprétations évoquées.

\section{$4 \quad$ L'analyse}

Jusqu'ici, nous avons vu que les règles de transformation comme (8) ne couvrent pas les données essentielles impliquant en. L'analyse que nous proposons ci-dessous suggère que c'est parce que ces règles se concentrent sur les formes morphologiques concernées par ces règles sans tenir compte des informations essentielles fournies par le discours.

Comme nous l'avons mentionné dans notre discussion de (13), (14) et (16), les pronoms en gras dans je les ai vus aussi, j'en ai vu aussi et j'en ai pris aussi renvoient à des interprétations différentes. Selon nous, on peut expliquer cela de manière simple, sans introduire de notions théoriques complexes. Ce que nous proposons, c'est que l'apprenant a besoin de comprendre certains aspects de la notion de référence, car cela lui permettra de différencier les trois contextes. Cela signifie que l'apprenant doit savoir ce que le pronom désigne, c'est-à-dire ce qu'il représente : en (13), les représente tous les biscuits vus par Jean (et par Marie), en (14), en représente quelques biscuits que Marie a vus mais en aucune manière les mêmes biscuits que Jean a vus et en (16), en représente une sous-partie des biscuits mentionnés par Jean. Autrement dit, dans le premier cas, nous avons affaire à une référence définie - nous nous référons à l'ensemble des biscuits qui ont déjà été introduits dans le discours -, dans le second cas, il s'agit d'une référence indéfinie - nous nous référons à des biscuits qui n'ont pas été mentionnés - et dans le troisième cas, nous sommes confrontés à une partie d'une référence déjà mentionnée, il est donc question d'une interprétation partitive. L'idée est que la référence définie représente un ensemble donné, que la référence indéfinie représente un autre ensemble, tandis que le partitif représente une partie d'un ensemble donné.

La même analyse s'applique à (17) et (18). En (18a), les renvoie à l'ensemble des pommes mentionnées par Jean (l'intégralité des 5 pommes), en (18b), en se rapporte à un ensemble différent de pommes, c'est-à-dire à des pommes n'ayant pas encore été introduites dans le discours (il y a 10 pommes au total) et en (18c), en se rapporte à une sous-partie des 5 pommes déjà mentionnées. En bref, en est utilisé lorsque la référence n'est pas définie (cf. Ihsane 2013).

En introduisant ces notions liées à la référence, on permettra à l'apprenant de faire la distinction entre les différentes interprétations décrites ci-dessus, et donc de choisir le bon pronom, en étant conscient des 
différents usages de ces pronoms. Comme le concept de référence concerne non seulement les exemples avec des, mais également ceux introduits par $d u$, de la, de $l^{\prime}$, qui peuvent être indéfinis ou partitifs (comme en (14) et (16) respectivement), il couvre toutes les formes qui peuvent être remplacées par en mentionnées dans (8a), à l'exception de de + nom nu. Ce n'est cependant pas un problème puisque ces constructions peuvent, d'après nous, être assimilées à des constructions impliquant un nombre (cf. (3)) ou une expression de quantité (cf. (4)), c'est-à-dire aux cas en (8b). Les exemples pertinents sont repris cidessous pour plus de commodité :

(19) Combien de viande veux-tu? J'en veux un peu. / * J'en veux. / * J'en veux une.

(20) Combien de livres veux-tu? * J'en veux un peu. / * J'en veux. / J'en veux deux.

(19) et (20) comprennent tous deux une question contenant combien. Cependant, ils diffèrent en ce que la réponse à cette question doit comporter respectivement une quantité (un peu) et un nombre (deux). Comme la traduction de combien en anglais le suggère, la distinction est due au type de nom suivant de: quand c'est un terme non-comptable ('how much'), une quantité est nécessaire alors que quand il s'agit d'un terme comptable ('how many'), un nombre est nécessaire. Viande est non-comptable, d'où l'apparition d'un peu dans (19) et livres est comptable, d'où la présence de deux en (20). Cela signifie que les contextes illustrés en (19) et (20) peuvent être assimilés à d'autres expressions avec un nombre / une quantité. La différence est que le nombre / la quantité n'est pas explicitement mentionné(e) dans les questions en (19) et (20). En conséquence, l'apprenant du français doit déduire cette information du contexte, en prenant en compte un indice crucial : le type de nom qui est utilisé dans la question, par exemple. Cela implique que l'apprenant soit conscient du contraste entre comptable et non-comptable. Comme ce contraste se résume à savoir si nous avons affaire à des entités individualisées ou non (respectivement comptables vs. non-comptables), cela signifie que ce que nous devons savoir, c'est si la référence des expressions qui nous intéressent peut être divisée en entités ou pas. C'est ainsi que les questions concernant (19) et (20) sont liées au premier problème dont nous avons discuté, à savoir les différents usages des constituants avec des : c'est une question de référence.

La question suivante qui nous intéresse est de savoir si la discussion ci-dessus s'étend à de + nom nu dans des contextes négatifs comme (7), répété ci-dessous (21).

(21) Jean n'a pas de chien. Il n'en a pas.

Rappelons que nous avons signalé ci-dessus que ces constructions se distinguent des contextes avec combien, discutés dans (19) et (20), en ce qu'il n'y a pas de nombre / quantité qui apparaît avec en. Ce que nous proposons, c'est que les exemples avec la négation sont en fait similaires à des contextes comprenant des quantités comme beaucoup ou peu, sauf que la quantité n'est pas mentionnée explicitement (Ihsane 2013). Ceci est illustré en (22)-(24) :

$\begin{array}{llllll}\text { (22) Jean a } & \text { beaucoup } & \text { de livres. } & \text { Il en a } & \text { beaucoup. } & \\ \text { (23) Jean a } & \text { peu } & \text { de livres. } & \text { Il en a } & \text { peu. } & \\ \text { (24) Jean n'a pas } & \varnothing & \text { de livres. } & \text { Il n'en a } & \varnothing & \text { pas. }\end{array}$


Dans les exemples ci-dessus, en remplace de livres. Les quantités beaucoup et peu sont répétées dans les phrases contenant en. L'idée est que, de la même manière, $\varnothing$, qui est une quantité nulle, est présent dans la phrase avec en, mais comme cette quantité n'est pas explicite le parallèle doit être expliqué aux apprenants de français. Ce qui est intéressant dans (22)-(24) est que beaucoup / peu / Ø sont possibles à la fois avec des termes comptables (beaucoup / peu / pas de livre(s)) et non-comptables (beaucoup / peu / pas de lait). La différence entre beaucoup / peu et $\varnothing$ de lait est que la quantité $\varnothing$ est limitée à des contextes négatifs (Kayne 1981). Le fait qu'il y ait une certaine quantité exprimée par (24) est clair dans la traduction anglaise qui contient any, la contrepartie négative de some. Rendre le parallèle entre (22)-(24) explicite est sans aucun doute utile pour l'apprenant.

La discussion de (22)-(24) appuie aussi ce que nous suggérions plus haut dans cette section, à savoir le fait que en est utilisé lorsque la référence n'est pas définie : de livres ne se réfère pas à des livres introduits dans le discours mais précise simplement le concept pertinent, à savoir des livres plutôt que des magazines, par exemple. Ceci est également confirmé par des exemples tels que (25), où en peut être utilisé même en l'absence de coréférence. ${ }^{4}$

(25) J'aime bien ton jean, j'en voudrais un aussi.

Dans cet exemple, en ne représente pas ton jean mais plutôt le type de jean. La phrase pourrait parfaitement continuer ainsi : j'en voudrais un aussi, de jean comme le tien.

L'importance décisive de cette notion de référence dans l'utilisation de en est en outre étayée par les phénomènes d'accord. Prenons les exemples ci-dessous :

(26) Jean a mis des fourchettes dans le tiroir. Marie les a prises puis remises

dans le tiroir.

(27) Jean a mis des fourchettes dans le tiroir. Marie en a mis / * mises aussi.

En (26), les représente les fourchettes déjà introduites dans le discours, c'est-à-dire une référence définie, et les participes passés prises 'fém.pl' et remises 'fém.pl' sont accordés avec ce pronom, puisqu'il s'agit d'un complément d'objet direct qui précède les participes. En (27), en revanche, il n'y a pas d'accord sur le participe passé mis. Ce n'est pas surprenant puisque l'accord se fait en principe avec une référence définie et qu'en ne peut pas être utilisé avec une telle référence, comme indiqué ci-dessus. ${ }^{5}$ En d'autres termes, les phénomènes d'accord rapportés dans les exemples ci-dessus appuient l'analyse que nous proposons. L'absence d'accord avec en au passé composé est mentionnée dans un seul des manuels examinés dans le présent article, à savoir Valette et Valette (1989).

Les principales idées avancées ci-dessus peuvent être résumées comme en (28) : 


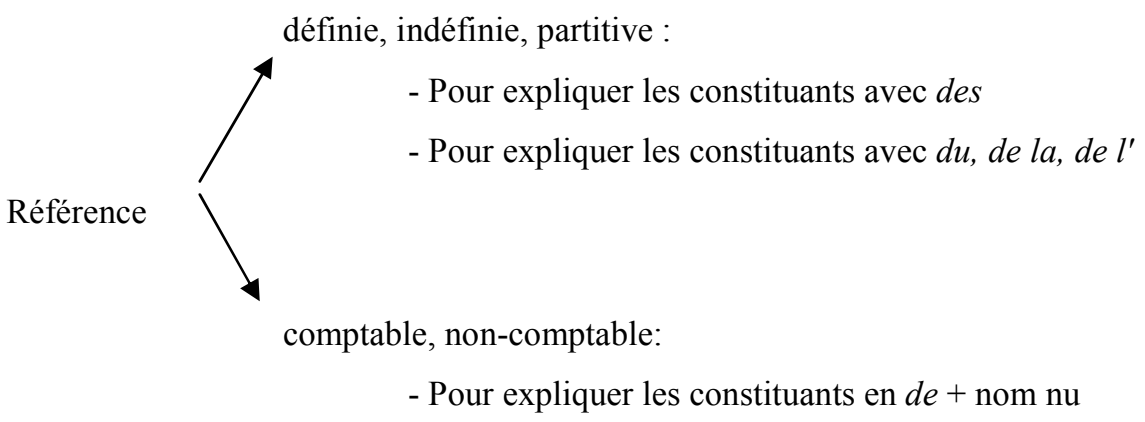

Le schéma en (28) montre que les notions liées à la référence pertinentes pour enseigner en se déclinent en degré de définition et en termes de comptabilité. En matière de définition, on distingue entre défini, indéfini et partitif. Cela permet non seulement d'expliquer comment en s'utilise dans des contextes avec des, mais également avec des constituants en $d u$, de la, de l'. Pour l'autre choix, on trouve une seule opposition, entre comptable et non-comptable. Cette différenciation permet d'expliquer comment utiliser en dans les contextes avec $d e+$ nom nu.

La formalisation en (28) diffère des règles de substitution telles que (8) en ce qu'elle ne concerne pas des formes morphologiques mais des informations fournies par le contexte d'énonciation. L'introduction de ces notions liées à la référence dans les grammaires ou les manuels pour les apprenants du français n'est pas une complication majeure. Ceci parce que la plupart des apprenants du français doit se familiariser avec la relation partie-tout, avec la distinction comptable/non-comptable et avec les notions défini/indéfini, puisque la compréhension de ces notions est nécessaire, notamment pour utiliser les déterminants correctement. Les manuels peuvent donc renvoyer le lecteur vers les unités concernées et/ou utiliser la discussion autour de en pour montrer que ces notions sont liées, précisément à travers le concept de référence. Quelques précisions concernant la terminologie pourraient être nécessaires (référence vs. référent, référence définie $=$ introduite dans le discours...). Elles peuvent être intégrées dans la discussion des exemples avec des ou de + nom nu.

\section{D'autres questions liées à l'enseignement de en}

Pour que le matériel d'enseignement sur en soit efficace, certains autres points de discussion pertinents pour les apprenants de français devraient être mentionnés dans les manuels d'enseignement. L'un d'eux est la position de ce pronom dans la phrase. D'autres questions concernent spécifiquement les apprenants anglophones du français, du fait que l'anglais ne possède pas toujours une contrepartie évidente à en.

La position de en dans la phrase n'est mentionnée que dans deux des manuels que nous avons examinés (Valette et Valette et Mitschke et Tano) : en, comme les autres pronoms clitiques, précède immédiatement le verbe / auxiliaire (cf. par exemple (22)-(24), (26)-(27)). Cela signifie que dans des contextes négatifs en suit la particule négative $n e$ (cf. (21)). En français oral, cependant, la particule négative ne est généralement omise (29). En conséquence, la position de en est entre le sujet et le verbe / auxiliaire (30).

(29) Je les vois pas. (vs. Je ne les vois pas.)

(30) J'en ai pas. (vs. Je n'en ai pas.) 
Les problèmes mentionnés ci-dessus sont pertinents pour tout apprenant de la langue française. En outre, certains aspects de l'utilisation de en sont particulièrement difficiles pour les apprenants de langue anglaise. Le principal problème est que, dans certains exemples en a une contrepartie en anglais (some/any) (cf. par exemple (9) et (24)) alors que dans d'autres, ce n'est pas le cas (cf. (22) et (23)). Ces exemples sont repris ci-dessous pour plus de commodité :

(31) Jean a acheté des livres. Il en a acheté. = (9)

(32) Jean n'a pas de livres. Il n'en a pas. $=(24)$

(33) Jean a beaucoup de livres. Il en a beaucoup. $=(22)$

(34) Jean a peu de livres. Il en a peu. $=(23)$

Il en a acheté en (31) correspond à He bought some, et Il n'en a pas en (32) à He doesn't have any, alors que Il en a beaucoup en (33) se traduit par He has many et Il en a peu en (34) par He has few, où many et few correspondent respectivement à beaucoup et peu. ${ }^{6}$ Des exemples comme (33) et (34) sont particulièrement difficiles pour les anglophones, qui souvent omettent en dans de tels contextes. (35) est une illustration d'une erreur réelle, typique pour un apprenant de langue anglaise.

(35) J'ai une. (avec pour intention : J'en ai une.)

Cette erreur peut être mise sur le compte de l'influence de l'anglais : le sens véhiculé par les phrases en français se traduit en anglais sans élément correspondant à en. D'où l'importance de mettre en évidence la différence entre les phrases (31)-(32) d'une part et (33)-(34) d'autre part : dans ces dernières, à savoir les constructions avec un nombre/une quantité, en est requis en français, en plus du nombre/de la quantité, contrairement à l'anglais.

Un autre fait auquel les anglophones se doivent de prêter attention est que some, en anglais, fonctionne à la fois comme déterminant et comme pronom, comme le montre la traduction de (31) : John bought some books. He bought some. Le français, en revanche, utilise deux éléments différents, des (déterminant) et en (pronom). La difficulté liée à ce point est qu'une locution avec des ne correspond pas toujours à en: souvenez-vous qu'un tel composant peut être pronominalisé par les (13). En outre, des doit apparaître avec des noms comptables, ce qui contraste avec some, qui est grammatical à la fois avec des termes comptables et non-comptables. Cela signifie que le déterminant some ne correspond pas toujours à des. C'est pourquoi l'apprenant doit être conscient des différences.

\section{Conclusion}

Dans cet article, nous avons montré que les règles de substitution fournies par les manuels destinés aux apprenants de français sont limitées. Elles le sont car elles fonctionnent comme des raccourcis, ignorant divers aspects dans leur description des faits.

Ce que nous avons proposé de faire est de s'éloigner des règles qui mettent l'accent sur les formes morphologiques, à savoir les segments de phrases, et de prendre en compte le contexte de l'énoncé. Cette 
dimension de la signification doit être considérée dans la description des faits, et donc rendue explicite à l'apprenant dans les manuels d'enseignement. Le but n'est pas de décrire tous les aspects touchant à en, mais plutôt de prendre en compte, pour chaque niveau, le contexte dans lequel en est utilisé pour que l'apprenant puisse avoir une meilleure compréhension de ce qui est en jeu. Ceci peut être réalisé par la sensibilisation de l'apprenant à des concepts plus larges comme celui de référence.

Notre analyse est basée sur l'étude de en. En particulier, nous avons montré que l'introduction de notions liées à la référence résout plusieurs problèmes qui ne sont pas traités par les règles transformationnelles. Cela implique de prendre en compte la distinction comptable/non-comptable, le rapport entre la partie et le tout (partitivité), la différence entre les éléments introduits dans le discours (définis) et les éléments non introduits dans le discours (indéfinis).

En conclusion, nous montrons que les règles mécaniques négligent des faits essentiels, et proposons que le contexte dans lequel un exemple / un énoncé est prononcé devrait être pris en compte dans les manuels. Il serait intéressant dans un travail futur de mener une étude de corpus pour vérifier si notre analyse se vérifie.

\section{Références bibliographiques}

Abry, D. et M.-L. Chalaron. (2003). La grammaire des premiers temps, Vol. 2. Clupguide.

Bartning, I. et S. Schlyter. (2004). Itinéraires acquisitionnels et stades de développement en français L2. Journal of French Language Studies 14: 281-299. Cambrige, Cambrige University Press.

Ihsane, T. (2013). En Pronominalisation in French and the Structure of Nominal Expressions. Syntax 16.3: 217-249.

Ihsane, T., C. Forel et F. Kusseling. (à paraître). Taking discourse into account: The limits of substitution rules in the treatment of the pronoun 'en'. Linguistica Atlantica 33 (1).

Mitschke, C. (2008). Imaginez: Le Français sans Frontières. Cours de Français Intermédiaire. Boston: Vista Higher Learning.

Mitschke, C. et C. Tano. (2010). Espaces. $2^{\text {nd }}$ ed. Boston: Vista Higher Learning.

Mitschke, C. et C. Tano. (2010). Promenades à travers le Monde Francophone. $\sigma^{\text {th }}$ ed. Boston: Vista Higher Learning.

Oates, M. D. et J. F. Dubois. (2010). Personnages: An Intermediate Course in French Language and Francophone Culture. $4^{\text {th }}$ ed. Hoboken: Wiley.

Ollivier, J. et M. Beaudoin. (2008). Grammaire Française, Quatrième Édition. Canada: Thomson Nelson.

Terrell, T. D., M. B. Rogers, B. J. Kerr et G. Spielmann. (2008). Deux Mondes: A Communicative Approach. $6^{\text {th }}$ ed. Boston: McGraw-Hill Higher Education.

Thompson, C. P. et B. G. Hirsch. (2003). Ensuite. $4^{\text {th }}$ ed. Boston: McGraw Hill.

Valette, J.-P. et R. M. Valette. (1989). Contacts: Langue et Culture Françaises. $4^{\text {th }}$ ed. Boston: Houghton Mifflin.

\footnotetext{
${ }^{1}$ Nous remercions les organisateurs du 4e Congrès Mondial de Linguistique Française (cmlf2014) de nous permettre de publier ce travail, ainsi que deux relecteurs anonymes pour leurs suggestions et remarques. Une version différente de ce papier va paraître dans Linguistica Atlantica 33 (1) en anglais.
} 


\footnotetext{
${ }^{2}$ Nous nous en sommes tenus à ces ouvrages-là parce l'une des auteures les utilise pour enseigner le français à des étudiants universitaires anglophones aux États-Unis. Nous remercions un relecteur qui nous signale des ouvrages de grammaire du FLE publiés en France, en particulier Abry et Chalaron (2003), avec une progression intéressante dans l'enseignement du système de pronoms entre le volume 1 pour débutants et le volume 2, et un autre relecteur qui mentionne Bartning et Schlyter (2004).

${ }^{3}$ Les tours idiomatiques tels que $s$ 'en aller ou ne pas en revenir (comme dans : Je n'en reviens pas!) ne concernent pas notre discussion puisqu'ils ne résultent pas de l'application mécanique de règles telles que celles présentées en (8) mais sont des expressions figées.

${ }^{4}$ Nous remercions un relecteur anonyme pour cette remarque et cet exemple.

${ }^{5}$ Par 'référence définie' nous ne nous limitons pas aux exemples avec un article défini. Une référence peut être définie par une relative (nous remercions un relecteur d'avoir attiré notre attention sur ce point) :

(i) C'est une lointaine cousine que je n'ai jamais rencontrée. Nous en avions pourtant rencontré plusieurs à l'enterrement de notre grand-oncle.

${ }^{6}$ Un relecteur observe que l'apprenant doit être sensibilisé à l'ordre des mots en français puisque en et some n'occupent pas la même position : J'en ai vs. I have some. La même observation vaut pour les pronoms personnels comme dans Je les veux par opposition à I want them.
} 